\title{
FRACTIONAL DERIVATIVES OF HOLOMORPHIC FUNCTIONS ON BOUNDED SYMMETRIC DOMAINS OF $\boldsymbol{C}^{n}$
}

\author{
ZENGJIAN LOU \\ Department of Mathematics \\ Qufu Normal University \\ Qufu Shandong, 273165 \\ P R CHINA
}

(Received March 8, 1994 and in revised form January 13,1995)

$$
\begin{aligned}
& \text { ABSTRACT. Let } f \in H\left(B_{n}\right) \quad f^{[\beta]} \text { denotes the } \beta \text { th fractional derivative of } f \text { If } \\
& f^{[\beta]} \in A^{p, q, \alpha}\left(B_{n}\right) \text {, we show that } \\
& \text { (I) If } \beta<\frac{\alpha+1}{p}+\frac{n}{q}=\delta \text {, then } f \in A^{s, t, \alpha}\left(B_{n}\right) \text {, and }\|f\|_{s, t, \alpha} \leq C\left\|f^{[\beta]}\right\|_{p, q, \alpha}, s=\frac{\delta p}{\delta-\beta}, t=\frac{\delta q}{\delta-\beta} \\
& \text { (II) If } \beta=\frac{\alpha+1}{p}+\frac{n}{q} \text {, then } f \in B\left(B_{n}\right) \text { and }\|f\|_{B} \leq C\left\|f^{[\beta]}\right\|_{p, q, \alpha} \\
& \text { (III) If } \beta>\frac{\alpha+1}{p}+\frac{n}{q} \text {, then } f \in \wedge_{\beta-\frac{\alpha+1}{p}-\frac{n}{q}}\left(B_{n}\right) \text { especially If } \beta=1 \text { then } \\
& \|f\|_{\wedge_{1-\frac{\alpha+1}{p}-\frac{n}{q}} \leq C\left\|f^{[1]}\right\|_{p, q, \alpha} \text { where } B_{n} \text { is the unit ball of } C^{n}}
\end{aligned}
$$

KEY WORDS AND PHRASES. Fractional derivative, Bergman space, Bloch space, Lipschitz space 1991 AMS SUBJECT CLASSIFICATION CODE. 32A10

Let $\Omega$ be a bounded symmetric domain in the complex vector space $C^{n}, o \in \Omega$, with BergmanSilov boundary $b, \Gamma$ the group of holomorphic automorphisms of $\Omega$ and $\Gamma_{0}$ its isotropy group. It is known that $\Omega$ is circular and star-shaped with respect to $o$ and $b$ is circular. The group $\Gamma_{0}$ is transitive on $b$ and $b$ has a unique normalized $\Gamma_{0}$-invariant measure $\sigma$ with $\sigma(b)=1$. Hua [2] constructed by group representation theory a system $\left\{\phi_{k v}\right\}$ of homogeneous polynomials, $k=0,1, \ldots$, $v=1, \ldots, m_{k}, m_{k}=\left(\begin{array}{c}n+k-1 \\ k\end{array}\right)$, complete and orthogonal on $\Omega$ and orthonormal on $b$.

By $H(\Omega)$ we denote the class of all holomorphic functions on $\Omega$. Every $f \in H(\Omega)$ has a series expansion

$$
f(z)=\sum_{k, v} a_{k v} \phi_{k v}(z), \quad a_{k v}=\lim _{r \rightarrow 1} \int_{b} f(r \xi) \overline{\phi_{k v}(\xi)} d \sigma(\xi)
$$

where $\sum_{k, v}=\sum_{k=0}^{\infty} \sum_{v=1}^{m_{k}}$ and the convergence is uniform on a compact subset of $\Omega$.

Let $f \in H(\Omega)$ with the expansion (0) and $\beta>0$. The $\beta$ th fractional derivatives of $f$ are defined, respectively, by

$$
\begin{aligned}
f^{[\beta]}(z) & =\sum_{k, v} \frac{\Gamma(k+1+\beta)}{\Gamma(k+1)} a_{k v} \phi_{k v}(z) \\
f_{[\beta]}(z) & =\sum_{k, v} \frac{\Gamma(k+1)}{\Gamma(k+1+\beta)} a_{k v} \phi_{k v}(z)
\end{aligned}
$$

It is known that $f^{[\beta]}, f_{[\beta]} \in H(\Omega)$ and

$$
f(r \xi)=\frac{1}{\Gamma(\beta)} \int_{0}^{1}(1-\rho)^{\beta-1} f^{[\beta]}(r \rho \xi) d \rho .
$$

Let $f \in H(\Omega)$. It will be said that $f$ belongs to the Bergman spaces $A^{p, q, \alpha}(\Omega), 0<p, q \leq \infty, \alpha>-1$ if

$$
\|f\|_{p, q, \alpha}=\left\{\begin{array}{cc}
\left(\int_{0}^{1}(1-r)^{\alpha} M_{q}(r, f)^{p} d r\right)^{\frac{1}{p}}, & p<\infty \\
\sup _{0<r<1}(1-r)^{\alpha} M_{q}(r, f), & p=\infty
\end{array}\right.
$$

is finite, where 
and

$$
M_{q}(r, f)=\left(\int_{b}|f(r \xi)|^{q} d \sigma(\xi)^{q}\right), \quad 0<q<\infty
$$

$$
M_{\infty}(r, f)=\sup _{\xi \in b}|f(r \xi)|
$$

see $[1,3,5,6,7]$ for more on $A^{p . q . \alpha}(\Omega)$ For $0<p \leq \infty$, let $A^{p}(\Omega)$ denote $A^{p . p, o}(\Omega)$ (see $\left.[10,12]\right), H^{p}(\Omega)$ denote $A^{\infty, p, 0}(\Omega)$ (see [9])

Let $B_{n}$ denote the unit ball in $C^{n}$ A function $f \in H\left(B_{n}\right)$ is called a Bloch function, that is $f \in B\left(B_{n}\right)$, if $([8,11])$

$$
\|f\|_{B}=\sup _{z \in B_{n}}(1-|z|)\left|f^{[1]}(z)\right|<\infty
$$

For $0<\alpha<\infty$, the defintion of Lipschitz space $\wedge_{\alpha}\left(B_{n}\right)$ can be found in $[4, \S 88]$

In [10] and [12], Watanable and Stojan considered the problem If $f^{\prime} \in A^{p}(D)(D$ is the unit disc of $C^{1}$ ), then $q=$ ? such that $f \in A^{q}(D) \quad$ In this paper we consider and solve the same problem in $A^{p, q, \alpha}(\Omega)$

The main results of this paper are the following

THEOREM 1. Let $0<p, q \leq \infty, \alpha>-1,0<\beta<\delta \leq \frac{\alpha+1}{p}+\frac{n}{q}$, if $f^{[\beta]} \in A^{p, q, \alpha}(\Omega)$ and $f^{[\beta]}(r \xi)=O\left(\left\|f^{[\beta]}\right\|_{p, q, \alpha}(1-r)^{-\delta}\right)$, then $f \in A^{s, t, \alpha}(\Omega) \quad$ and $\quad\|f\|_{s, t, \alpha} \leq C\left\|f^{[\beta]}\right\|_{p, q, \alpha}$, where $s=\frac{\delta p}{\delta-\beta}, t=\frac{\delta q}{\delta-\beta}$

THEOREM 2. Let $0<p, q \leq \infty, \alpha>-1,0<\beta<\infty, f^{[\beta]} \in A^{p, q, \alpha}\left(B_{n}\right)$. same as above

(I) If $\beta<\frac{\alpha+1}{p}+\frac{n}{q}=\delta$, then $f \in A^{s, t, \alpha}\left(B_{n}\right)$, and $\|f\|_{s, t, \alpha} \leq C\left\|f^{[\beta]}\right\|_{p, q, \alpha}$, where $s, t$ are the

(II) If $\beta=\frac{\alpha+1}{p}+\frac{n}{q}$, then $f \in B\left(B_{n}\right)$ and $\|f\|_{B} \leq C\left\|f^{[\beta]}\right\|_{p, q, \alpha}$

(III) If $\beta>\frac{\alpha+1}{p}+\frac{n}{q}$, then $f \in \wedge_{\beta-\frac{\alpha+1}{p}-\frac{n}{q}}\left(B_{n}\right)$, especially If $\beta=1$, then $\|f\|_{\wedge_{1, \frac{\alpha+1}{p}-\frac{n}{q}}} \leq C\left\|f^{[1]}\right\|_{p, q, \alpha}$

REMARK. (i) Theorem 2(I) $(p=q, \alpha=0, \beta=n=1)$ extends the results of Watanable's and Stojan's (ii) Theorem $1(p=\infty)$ extends the results of Shi's ([9]) and Lou's $([6,7])$

\section{REFERENCES}

[1] AHERN, P AND JEVTIC, M, Duality and multipliers for mixed norm spaces, Mich. Math. J., 30 (1983), 53-63

[2] HUA, L K, Harmonic analysis of functions of several variables in the classical domains, Trans. Amer. Math. Soc., 6 (1963).

[3] JEVTIC, M, Projection theorems, fractional derivatives and inclusion theorems for mixed-norm spaces on the ball, Analysis, 9 (1989), 83-105

[4] KRANTZ, S.G, Function Theory of Several Complex Varıables, John Wiley \& Sons, New York, 1982

[5] LOU, Z J, A note on a conjecture of S Axler, J. Math. Res. \& Exp., 11 (4) (1991), 629-630 (Chinese)

[6] LOU, Z J, Hardy-Littlewood type theorem on Bergman spaces, J. Math. Res. \& Exp., 11 (3) (1991) (Chinese)

[7] LOU, Z J , Hardy-Littlewood type theorem on Bergman spaces of several complex variables, $J$. Qufu Normal Unlv., 1 (1992), 111-112 (Chinese)

[8] LOU, Z J., Characterizations of Bloch functions on the unit ball of $\mathrm{C}^{\mathrm{n}}$, Kodal Math. J., 16 (1993), 74-78

[9] SHI, J H, Hardy-Littlewood theorems on bounded symmetric domains, Science in China, 4 (1988), 366-375

[10] STOJAN, D, Some new properties of the spaces $A^{p}$, Mat. Vesinc, 5 18,33 (1981), 151-157

[11] TIMONEY, R M , Bloch functions in several complex variables, Bull. London. Math. Soc., 12 (1980), 241-267 


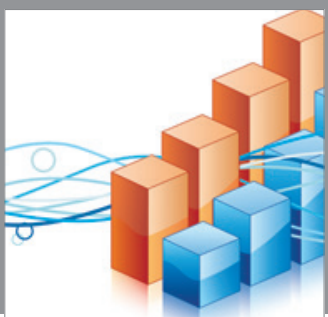

Advances in

Operations Research

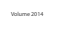

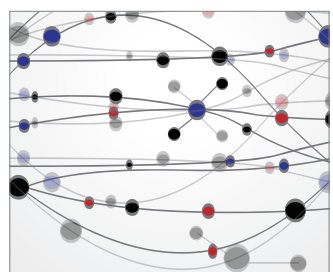

\section{The Scientific} World Journal
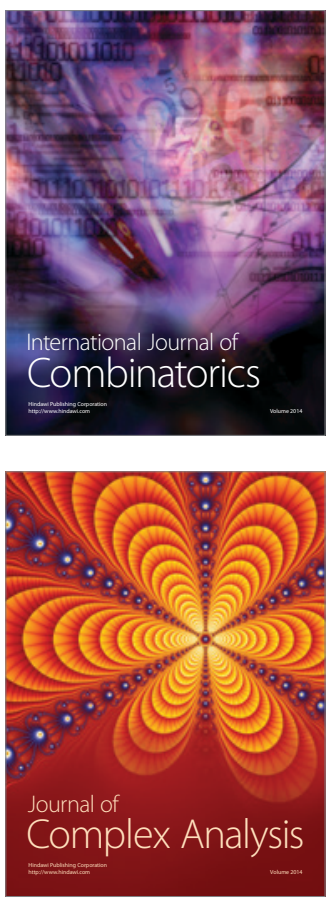

International Journal of

Mathematics and

Mathematical

Sciences
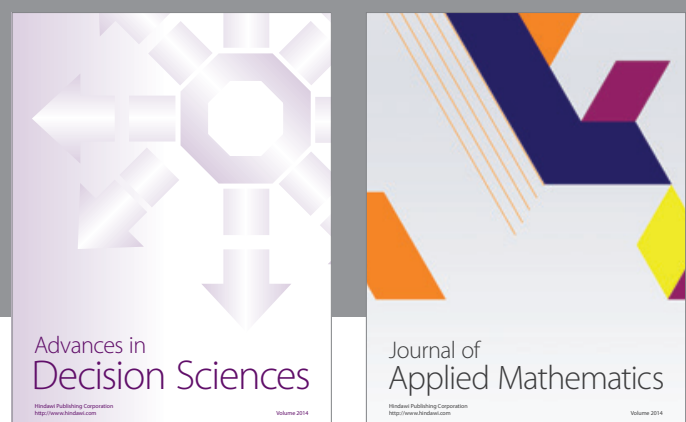

Journal of

Applied Mathematics
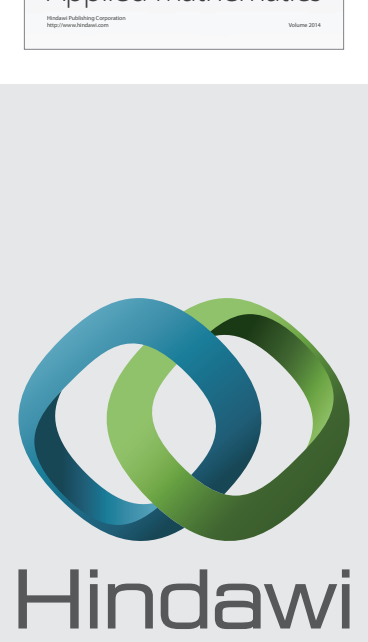

Submit your manuscripts at http://www.hindawi.com
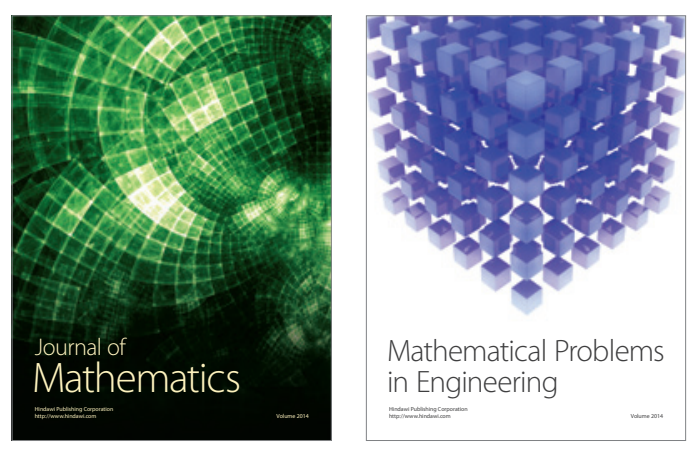

Mathematical Problems in Engineering
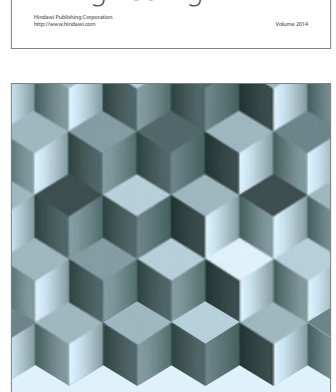

Journal of

Function Spaces
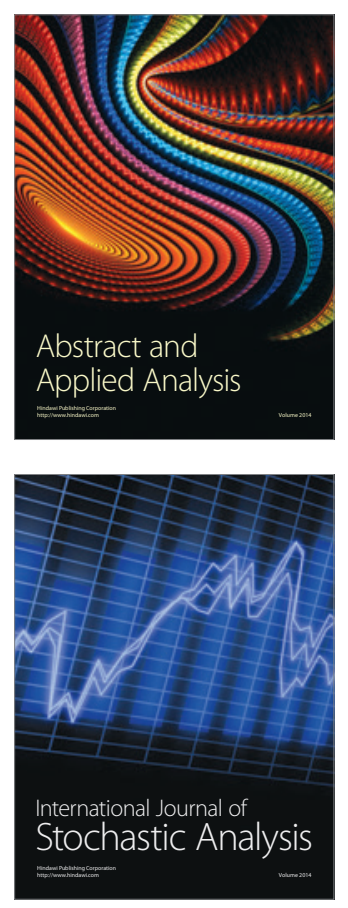

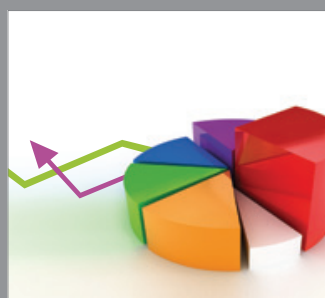

ournal of

Probability and Statistics

Promensencen
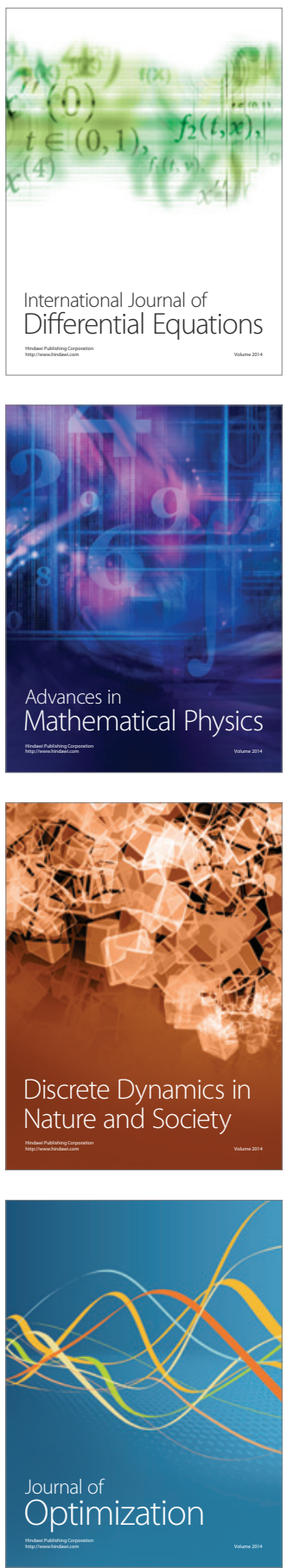